\section{SuperLab: General-purpose Macintosh software for human experimental psychology and psychological testing}

\author{
JAMES V. HAXBY \\ Laboratory of Neurosciences \\ National Institute on Aging, Bethesda, Maryland \\ RAJA PARASURAMAN \\ Catholic University, Washington, D.C. \\ FRANÇOIS LALONDE \\ Laboratory of Clinical Science \\ National Institute of Mental Health \\ Bethesda, Maryland \\ and \\ HISHAM ABBOUD \\ Cedrus Corporation, Wheaton, Maryland
}

SuperLab is a general-purpose psychology testing package for the Macintosh. SuperLab presents static visual and auditory stimuli in blocks of trials, each trial consisting of a user-specified sequence of stimuli. Responses can be recorded from the keyboard or from switches connected to an I/O board. Stimuli can be contingent on subjects' responses, allowing feedback based on response accuracy. Timing uses Time Manager routines from the Macintosh Toolbox. Data are recorded in a text format with tabs delimiting fields, allowing analysis and presentation by other Macintosh spreadsheet, statistics, and graph-making applications. SuperLab has a Macintosh user interface for developing experiments. Psychological tasks can also be designed and modified with any application that generates a text format file.

Application programs on Macintosh computers use a common graphic user interface and are constrained by a strict set of guidelines for programmers. Consequently, all Macintosh applications have a common look and feel that greatly facilitates the learning of new programs. The Macintosh environment also stores data for generating a graphic display in a common format (PICT) used by most programs, greatly facilitating transfer of graphic information between programs. Macintosh users often use multiple programs when working on a single project, exploiting the special capabilities of each.

We decided to create a Macintosh program for developing and presenting cognitive tasks for psychology experiments and psychology testing. The principal goal of this project was to make it possible for us, as research psychologists, to use the Macintosh for computer-controlled experiments. Specifically, we wanted to use visual and

Correspondence should be addressed to J. V. Haxby, Laboratory of Neurosciences, NIA, Building 10, Room 6C414, National Institutes of Health, Bethesda, MD 20892 (e-mail: haxby@alw.nih.gov).

Note: R.P. and H.A. have a financial interest in SuperLab.-Editor auditory stimuli generated by other Macintosh applications. Another goal was to minimize the need for extensive programming for every new experiment or test. We attempted to devise a general-purpose structure for this program that could accommodate a wide range of experimental and testing paradigms. We hoped that the Macintosh graphic user interface would make it easier for new students and technicians to learn the program and allow nonprogrammers to develop computerized experimental tasks. The result of this project is SuperLab, Version 1.5. SuperLab can be run on any Macintosh computer with System 6.0.3 or later, including System 7.

\section{USING SUPERLAB IN THE MACINTOSH ENVIRONMENT}

SuperLab is designed to be used in conjunction with other Macintosh applications. SuperLab itself performs two main functions. First, SuperLab has a built-in editor for developing new experiments or tests. The file that stores the information that determines how the experiment or test will run is called a script. This part of SuperLab will be referred to as the Script Editor. Second, SuperLab has a program that first runs the experiment and then stores the response data in a file. This part of SuperLab will be referred to as the Runtime Program.

By design, SuperLab does not perform many of the functions necessary for developing a psychology experiment and analyzing the results. SuperLab is designed to read graphics and sound files generated by other applications and present them using the Runtime Program. The Runtime Program generates a response data file in text format that can be read into most statistics or mathematics applications for the analysis of results. SuperLab does not generate new graphics or sound files. SuperLab does not perform any calculations to score or analyze results.

\section{THE STRUCTURE OF AN EXPERIMENT IN SUPERLAB}

SuperLab has a very general structure that allows stimuli to be presented in any sequence specified by the experimenter, and it records responses on the keyboard or on other devices interfaced to a Macintosh computer through a National Instruments NB-DIO-24 I/O card. The sequence in which stimuli are presented are organized into blocks of trials, each trial being a sequence of events or stimuli (Figure 1). Stimulus duration can be specified within limitations imposed by the video display refresh cycle. Reaction time is measured as the interval between stimulus onset and response.

\section{Blocks}

SuperLab groups trials into blocks. Trials can be presented either in the user-specified order or in a randomized 


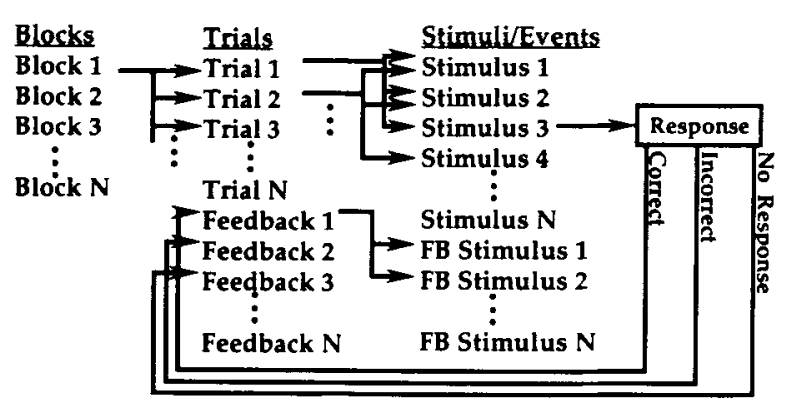

Figure 1. The structure of an experiment in SuperLab. Stimuli are organized into blocks of trials. Each trial is a sequence of stimuli, with no limit on the number of stimuli. Stimuli can be presented in multiple trials. Some trials can be designated feedback trials. The experimenter can specify which feedback trials are presented after a response. Different feedback trials can be presented after correct, incorrect, and no responses.

order. Trials designated as feedback trials are contingent on subjects' responses (see below).

\section{Trials}

Each trial consists of a sequence of stimuli. The user may specify the number of stimuli presented in each trial, which may vary from trial to trial. The same stimulus can be presented in several trials. SuperLab can record input from subjects on multiple events during a trial, allowing subject-paced experimental designs in which some responses indicate that the subject is ready to proceed with the rest of a trial.

In practice, the term trial has a more general meaning in SuperLab than it usually has in experimental psychology, referring as it does to any sequence of stimuli. In addition to trials used to obtain response data, trials can be used to present instructions or to indicate a pause between blocks of trials. We often present instructions for a block in the first trial and begin the experiment proper on the second trial. An even more unusual use of trials is for feedback, as described in the next section.

Feedback trials. Trials can be designated as feedback trials. Such a trial is a sequence of one or more stimuli presented only after a subject makes a response to a stimulus. For each stimulus event that allows a subject response, the experimenter can specify feedback trials that are presented if the subject makes a correct response, an incorrect response, or no response within a specified time limit. After a feedback trial has been presented, processing returns to the event following the one that called the feedback trial.

Trial codes. SuperLab allows the experimenter to define multiple codes that can be used to sort trials by type for data analysis. Codes are used to associate the values of an experiment's independent variables with each observation of a dependent variable. The experimenter may define up to 8 values for each code. We have used codes to indicate the difficulty level of trials (easy, moderate, hard), the validity of cues in a Posner shift of spatial at- tention task (valid, invalid), the degree of rotation in a mental rotation task $\left(0^{\circ}, 30^{\circ}, 60^{\circ}, 90^{\circ}\right.$, etc. $)$, and set size in a Treisman visual search task. The experimenter associates the value of each code with every trial when creating the experiment with the Script Editor (see Figure 2). The Runtime Program saves the value of each code on every response line for a trial in the data file, making it possible to use these codes as variables for sorting the data (see Figure 3).

\section{Stimuli/Events}

SuperLab can present three types of stimuli: visual stimuli on a computer monitor, auditory stimuli, and digital output to stimulus devices of the experimenter's design. The duration of visual and digital stimuli can be timed within limitations set by the refresh cycle on computer monitors. The duration of an auditory stimulus is set as the length of the sound file that stores that stimulus. SuperLab terminates the current stimulus and proceeds to the next according to one of three criteria. The stimulus can be terminated after a set time limit, after the subject makes a criterion response (either the correct response or any response), or after either a criterion response or, if no response is made, a set time limit. For each stimulus that accepts responses from the subject, the experimenter can specify feedback trials that are presented after the subject makes the correct, the incorrect, or no response. The dialog box used to specify information about stimulus presentation is shown in Figure 4.

Visual stimuli. SuperLab can present visual stimuli on any Macintosh monitor. The visual stimuli, which can be any PICT or PICT2 file generated by any Macintosh application, are stationary. These stimuli can be object-oriented drawings or pixel-mapped images, such as digitized drawings or photographs. On Macintosh computers without color capability, only object-oriented and bit-mapped drawings can be presented. On Macintosh II computers, SuperLab can only use the system look-up tables for color and gray-scale stimuli. If gray-scale photographs are used,

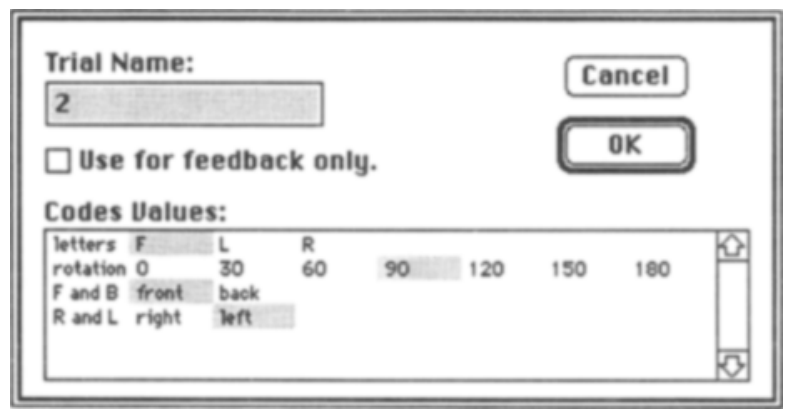

Figure 2. Dialog box for naming a trial and associating code values with that trial. This trial was taken from an experiment in which mental rotation speed was measured by asking subjects to indicate whether letters, presented at various degrees of rotation, were shown in normal or mirror-reversed orientation. On this trial, the letter $F$ was presented frontward, rotated to the left by $90^{\circ}$. 


\begin{tabular}{|c|c|c|c|c|c|c|c|c|c|c|}
\hline & $\bar{A}$ & $\mathbf{8}$ & C & D & $\bar{E}$ & $\mathbf{F}$ & $\mathbf{G}$ & $\mathrm{H}$ & 1 & $\mathbf{J}$ \\
\hline 1 & JHaxby & & & & ? & & & & & \\
\hline 2 & \multicolumn{2}{|c|}{ Letter Rotation } & & & 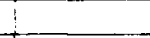 & & & & & \\
\hline 3 & $11 / 9 / 91$ & $17: 41$ & & & 1 & & & & & \\
\hline 4 & & & & & 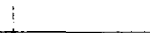 & & & & & \\
\hline 5 & Trial Name & Trial * & Event & Response & Error Code & Reaction Time & letters & rotation & $F$ and $B$ & $A$ and $L$ \\
\hline 6 & Instructions & 1 & 1 & B1 & $\mathrm{c}$ & 21916 & & & & \\
\hline 7 & 1. & 2 & 2 & B1 & $c$ & 780 & L & 150 & front & right \\
\hline 8 & 2 & 3 & 3 & 81 & C & 671 & $F$ & 90 & front & left \\
\hline 9 & 3 & 4 & 4 & 82 & $\mathrm{C}$ & 626 & $F$ & 30 & back & right \\
\hline 10 & 4 & 5 & 5 & 81 & $c$ & 846 & $R$ & 150 & front & left \\
\hline 11 & 5 & 6 & 6 & 81 & $E$ & 1295 & $L$ & 90 & back & right \\
\hline 12 & 5 & 6 & 6 & $B 2$ & $s c$ & 1623 & L & 90 & back & right \\
\hline 13 & 6 & 7 & 7 & B2 & $\mathrm{C}$ & 1327 & L & 180 & back & right \\
\hline 14 & 7 & 8 & 8 & $\mathrm{~B} 2$ & $\mathrm{ic}$ & 666 & $R$ & 60 & back & right \\
\hline 15 & 8 & 9 & 9 & B1 & C & 642 & $F$ & 30 & front & right \\
\hline 16 & 9 & 10 & 10 & B1 & $c$ & 545 & $L$ & 60 & front & left \\
\hline 17 & 10 & 11 & 11 & $B 2$ & C & 691 & R & 0 & back & right \\
\hline 18 & 11 & 12 & 12 & $B 1$ & C & 702 & $F$ & 120 & front & right \\
\hline 19 & & & & & & & & & & \\
\hline 20 & etc. & & & & & & & & & \\
\hline
\end{tabular}

Figure 3. Sample SuperLab data file generated by a script for measuring mental rotation speed. The file is presented as a Microsoft Excel table. The header contains the subject name, the name of the script, date, and time of day. Data lines indicate the trial and event (stimulus) during which responses were recorded, the nature of the response (Button 1 or Button 2 from the $\mathrm{I} / \mathrm{O}$ board), an error code (correct, error, self-correction), reaction time (in milliseconds), and user-defined trial codes indicating the letter presented, rotation of the letter in degrees, orientation of the letter (frontward or backward), and the direction of rotation.

it is recommended that the experimenter use the Macintosh Control Panel to switch to the gray-scale look-up table, because the system color look-up table has only 13 levels of gray. The visual stimuli can be centered on the screen or drawn at user-specified locations.

Several features of SuperLab allow the experimenter to synchronize timing with the refresh cycle of a monitor

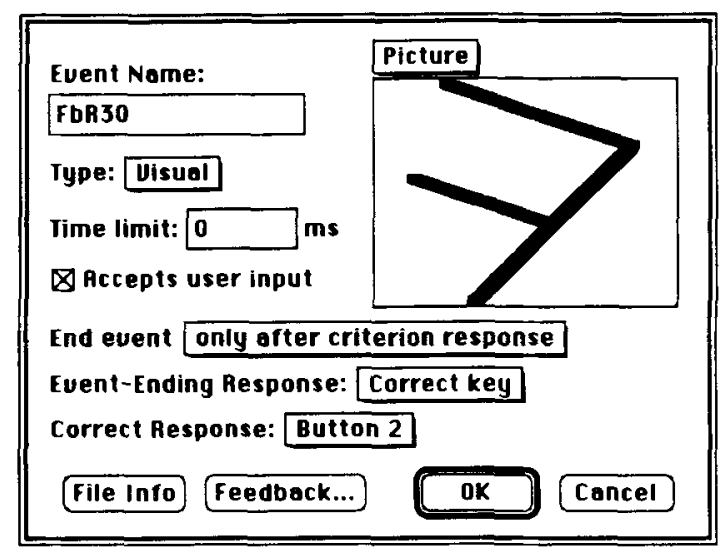

Figure 4. Dialog box for setting up a stimulus event. Pop-up menus allow the user to select stimulus type (visual, auditory, digital, or interstimulus interval), the criterion for ending the event (after a time limit, a response, or after whichever comes first), the response that can end the event, and the correct response. Another pop-up menu over the stimulus box changes for each stimulus type and allows the user to specify more options for stimulus presentation. Clicking the "Feedback..." button presents another dialog box for specifying feedback trials contingent on the subject's response. and to accommodate the length of time needed to draw a new stimulus to video memory. A relatively discrete visual stimulus onset can be achieved by choosing an instant switching option, under which the screen is kept blank while a new drawing or set of drawings is transferred into video memory. This is accomplished by setting all values of the look-up table equal to the background color, which can be specified by the experimenter. After drawing is completed, SuperLab waits until the next vertical blanking signal and changes back to the system lookup table. The complete stimulus is thus presented in a single refresh cycle. Multiple drawings can be transferred to video memory while the screen is blank, allowing the experimenter to construct stimulus arrays from multiple PICT files.

The instant switching option can only be used with color tables with 4, 16, or 256 colors. Macintoshes with black and white video only cannot use this option. On these machines, creating a large and complex visual stimulus can take substantially longer than a single refresh cycle, introducing considerable temporal imprecision. The instant switching option also cannot be used with 24-bit color images.

Visual stimulus durations are limited to integer multiples of the refresh cycle. The stimulus is terminated by switching to the blank screen look-up table during the first vertical blanking signal following the end of the stimulus interval, giving a discrete stimulus offset as well as stimulus onset. Response latencies are timed relative to the vertical blanking signal when the look-up table was switched. 
Because drawing time can add a variable interval between stimuli, SuperLab has a function that allows precise timing of the blank interval preceding a stimulus. SuperLab calculates the length of time needed for drawing a stimulus or array of stimuli and compares this interval to the user-specified prestimulus interval. If the drawing time is not too long, SuperLab keeps the screen blank for the user-specified prestimulus interval, which includes drawing time. As with all visual stimulus intervals, the prestimulus interval is limited to integer multiples of the monitor's refresh cycle.

If the experimenter does not want the screen to switch to blank between stimuli, he/she can choose not to use the instant switching option. In this case, stimulus onset and offset are less discrete, making the timing of responses less precise.

Auditory stimuli. SuperLab can present monaural or stereo sounds from files in SoundEdit format (Farallon Computing, Berkeley, CA). These sounds can be recorded at sampling frequencies of $5500,7400,11000$, and $22000 \mathrm{~Hz}$, with 8-bit numerical resolution for each sample. SuperLab allows some modulation of auditory stimulus frequency. Responses are timed relative to the onset of the auditory stimulus. Audio media boards that allow higher quality sound recording and presentation cannot be used with the current version of SuperLab.

Digital output stimuli. SuperLab can also present stimuli as digital output from eight ports of the National Instruments NB-DIO-24 I/O NuBus I/O board. This feature can be used by enterprising researchers who plan to build a device that could present a tactile stimulus or turn on a light in response to the digital output signal. For example, we have designed a reaction time device with lights inside of buttons that SuperLab can turn on and off.

Interstimulus interval. A fourth event type can be chosen that presents a blank interval of specified duration. The interstimulus interval (ISI) turns the screen blank by switching to the blank look-up table at the vertical blanking signal, allowing precise timing of responses to stimuli after the stimulus has been removed. Reaction times for responses made during an ISI must be calculated as the recorded reaction time plus the stimulus presentation interval. The stimulus presentation interval can be recorded as a trial code, simplifying calculation by making that value available in the data file on the same line as the reaction time.

\section{FILES USED OR CREATED BY SUPERLAB}

\section{Stimulus Files}

SuperLab can read PICT or PICT2 format graphics files for visual stimuli. The PICT and PICT2 format is a general format that can store graphic displays as object-oriented drawings or as pixel-mapped images and is available as an option for saving files in essentially all Macintosh graphics application programs. SuperLab can read SoundEdit format sound files for auditory stimuli. This is the format devised by Farallon Computing for use with their MacRecorder Sound System. The script file saves the names and locations of these files so that they are automatically read into RAM when the script file is opened. To avoid disturbing the timing of events during an experiment, no stimulus files are read during run time. An experiment that uses a large number of large visual files, such as pixel-mapped photographs, or large auditory files will be limited by the amount of RAM available on the computer being used.

\section{The SuperLab Script File}

The SuperLab script file contains all the information regarding how an experiment or test is to be run. With the SuperLab Script Editor's point-and-click interface, the experimenter sets up the experiment; the information is then stored in a tab-delimited text file. When the script file is opened, SuperLab reads the script file, translates it into the parameters for running the experiment, and reads all stimulus files into RAM.

The SuperLab script file was made a tab-delimited text file so that it could be read into other Macintosh applications for editing. Word processing applications, such as Microsoft Word (Microsoft, Redmond, WA), and spreadsheet applications, such as Microsoft Excel (Microsoft, Redmond, WA), are both well suited for some editing of script files. We have found that the SuperLab Script Editor provides the best method for initially developing a test or experiment, but that some modifications of the script are accomplished more easily with the use of other applications. We have used other Macintosh text editors to generate alternative forms of an experiment with trials presented in different orders, to change the length of presentation of stimuli, and to change the screen locations at which stimuli are presented.

\section{The SuperLab Data File}

The Runtime Program generates a data file in tabdelimited text format. A sample data file is presented in Figure 3. The data file has a brief header that contains the name of the subject, the name of the script for the experiment or test, and the date and time the experiment was run. Each data line contains information about one response. These data consist of the trial and event during which the response was made, the nature of the response, a code for the correctness of the response, the reaction time, and the user-specified codes that can be used to sort responses by trial type. The tab-delimited text format is generic; it can be read by essentially all Macintosh database and statistics applications.

\section{TIMING}

SuperLab has two timing options, referred to as Time Manager 1 and Time Manager 2, that use timers described in Volume 6 of Inside Macintosh (Apple Computer, 1991). The Time Manager routines used for these timers require System 6.0 .3 or later. Both timers have 
adequate temporal resolution and can accommodate long enough intervals for most psychology experiments and tests. One timer (Time Manager 1) ticks every millisecond; the other (Time Manager 2) ticks every $20 \mu \mathrm{sec}$. The maximum interval for one event is over 1 day for the Time Manager 1 timer and approximately $35 \mathrm{~min}$ for the Time Manager 2 timer.

\section{RESPONSE INPUT}

Superlab can record responses either from the keyboard or from devices that send a digital signal to the National Instruments NB-DIO-24 NuBus I/O card. SuperLab cannot record responses from the mouse or from analog input devices. Therefore, SuperLab cannot be used for most motor control and motor learning experiments. SuperLab treats each keystroke from the keyboard as a separate response. Consequently, multiple keystroke responses, such as words, create cumbersome data files that are difficult to manipulate.

Recording keyboard responses can be timed with two levels of temporal resolution. Using the standard Macintosh Toolbox call (GetNextEvent), response timing is accurate to within $16.67 \mathrm{msec}$. A second method uses an ADB Manager low-level access, as described in Volume 5 of Inside Macintosh (Apple Computer, 1986). Using Macintosh Time Manager routines, one of us (H.A.) has found that this access polls the keyboard approximately every $4 \mathrm{msec}$ on the Macintosh IIci. We have not tested the temporal precision of this routine on other Macintoshes.

SuperLab can also record responses through the serial ports. Each serial port can accommodate input from a single switch.

Input to the National Instruments NB-DIO-24 NuBus I/O card requires a simple circuit with a switch closure and a $10-k \Omega$ resistor connecting the $5-V$ power source and the appropriate digital input line. We have used this card for input from hand-held buttons, from buttons mounted in custom-made boxes, and from voice-activated relays. The National Instruments card allows access to input lines in $20 \mu \mathrm{sec}$ or less.

\section{LIMITATIONS OF SUPERLAB}

SuperLab was designed to have a very general structure that could accommodate a wide range of experimental and testing paradigms. It has limitations that restrict its use for many experimental paradigms. The limitations that we are aware of are described in this section to help the prospective user evaluate the suitability of SuperLab for his/her needs.

SuperLab does not perform any functions for generating new visual or auditory stimuli, because we doubted that we could improve on the capabilities available in other Macintosh applications. It also performs no numerical analyses of data. Existing Macintosh applications, such as JMP (SAS Institute, Cary, NC), Systat (Systat, Evanston, IL), and StatView (Abacus Concepts, Berkeley, CA) are adequate for this type of analysis. Moreover, analysis needs are too variable across experimental and testing paradigms to be accommodated with a reasonable number of canned procedures, and any attempt to meet these needs within SuperLab would detract from its generality.

SuperLab cannot present data trials in a sequence that is conditional on a subject's responses, precluding the use of SuperLab in studies of psychophysical thresholds that use a staircase method and in cognitive tests that use a branching item structure. A revision of SuperLab that will allow conditional branching of trial sequence is planned; this will be part of Version 2.0.

SuperLab presents only stationary visual stimuli, making it inappropriate for any visual or motor studies requiring moving stimuli.

SuperLab can only present auditory stimuli using Macintosh's built-in audio capability, which has a maximum sampling rate of $22 \mathrm{kHz}$ with 8 bits per sample. Users who wish to present higher quality sounds using audio media boards will not be able to use SuperLab for these experiments.

The timing of intervals between visual stimuli is complicated by the time necessary to draw a stimulus to video memory and by the refresh cycle of computer monitors. Gaining precision of timing over sequences of stimuli requires adjusting stimulus intervals for drawing time. This is done by SuperLab for blank intervals preceding a stimulus, but it must be calculated by the experimenter for more complicated sequences. A greater problem is the inability of SuperLab to record responses while a stimulus is being drawn to video memory, resulting in a significant period of deadtime. This shortcoming precludes presenting visual masks shortly after a stimulus. A revision of SuperLab is planned that will eliminate the dead-time problem.

SuperLab cannot record input from the mouse, from analog input devices, or from SCSI devices. It cannot, therefore, be used for most motor control and motor learning experiments. SuperLab can record only single-stroke responses from the keyboard, making it cumbersome for psychological tests in which subjects type whole words or phrases in as responses.

SuperLab cannot present any information on the screen that reflects a subject's performance, such as mean accuracy or reaction time, which precludes the use of this type of information for subject feedback.

\section{LIMITATIONS ASIDE}

SuperLab is a general-purpose software package that makes it possible for psychologists to use Macintosh computers for presenting a wide range of tasks to human subjects in the context of experimental psychology or psychological testing. Its generality and ease of use should 
reduce the time and effort needed to set up new experiments or tests. It also has potential for use in teaching experimental psychology.

Availability. SuperLab is copyrighted and available for sale from Cedrus Corporation, P.O. Box 8026, Silver Spring, MD 20907. A free demonstration disk can be obtained on request. The price for a single copy is U.S. $\$ 475$. A $20 \%$ educational discount is available.

\section{REFERENCES}

Apple Computer, INC. (1986). Inside Macintosh (Vol. 5). Reading, MA: Addison-Wesley.

APPle Computer, INC. (1991). Inside Macintosh (Vol. 6). Reading. MA: Addison-Wesley.

(Manuscript received September 28, 1992: revision accepted for publication April 20, 1993.) 